\title{
Qualidade de vida relacionada à disfunção do assoalho pélvico de gestantes dos sistemas público e privado
}

\author{
Quality of life related to pelvic floor dysfunction among pregnant women assisted in the \\ public and private health system
}

\section{Calidad de vida relacionada con la disfunción del piso pélvico en mujeres embarazadas atendidas en el sistema de salud público y privado}

Paula Renata Amorim Lessa Soares ${ }^{1 *}$, Samila Gomes Ribeiro ${ }^{1}$, Cinthia Gondim Pereira Calou², Raquel Alves de Oliveira ${ }^{1}$, Antônio José Lima de Araújo Junior ${ }^{1}$, Ana Karina Bezerra Pinheiro.

\section{RESUMO}

Objetivo: Comparar a qualidade de vida relacionada a disfunção do assoalho pélvico entre gestantes atendidas no sistema público e privado de saúde, bem como identificar a prevalência das disfunções do assoalho pélvico em gestantes de baixo risco atendidas nesses cenários. Métodos: Estudo transversal, com amostra de 261 gestantes e a coleta ocorreu de setembro a janeiro de 2015. Os participantes receberam um Termo de Consentimento e os princípios da Bioética foram respeitados. Resultados: Os dados evidenciaram que gestantes atendidas no sistema público tem quase 4 vezes mais chances de ter disfunção sexual, quando comparadas com gestantes da assistência privada. As gestantes da assistência privada tiveram melhor QVRS em todos os domínios. Embora gestantes do serviço privado sejam menos ativas sexualmente, os resultados demonstraram que essas gestantes tendem a ter menos disfunção sexual, com associação significativa em todos os domínios. Os domínios que apresentaram maior impacto negativo na ordem crescente de médias nos dois serviços foram semelhantes sendo: "Desejo", "Excitação", "Orgasmo", "Lubrificação", "Dor" e "Satisfação". Os domínios "Lubrificação" e "Dor" no serviço privado apresentaram médias idênticas. Conclusão: Conclui-se que gestantes acompanhadas no serviço privado apresentaram melhores índices de qualidade de vida de forma geral, associado à disfunção sexual e incontinência urinária.

Palavras-chave: Qualidade de vida, Diafragma da pelve, Gravidez, Enfermagem.

\begin{abstract}
Objective: To compare the quality of life related to pelvic floor dysfunction among pregnant women assisted in the public and private health system, as well as to identify the prevalence of pelvic floor dysfunction in low-risk pregnant women assisted in these scenarios. Methods: Cross-sectional study with a sample of 261 pregnant women and the collection occurred from September to January 2015. Participants received a consent form and the principles of bioethics were respected. Results: The data showed that pregnant women attended in the public system are almost 4 times more likely to have sexual dysfunction when compared with pregnant women in private care. Pregnant women in private care had better hrqol in all domains. Although pregnant women from the private service are less sexually active, the results showed that these women tend to have less sexual dysfunction, with significant association in all domains. The domains that had the greatest negative impact on the increasing order of averages in the two services were similar, being: "desire", "arousal", "orgimento", "lubrication", "pain" and "satisfaction". The domains "lubrication" and "pain" in the private service presented identical means. Conclusion: It is concluded that pregnant women followed in the private service had better indexes of quality of life in general, associated with sexual dysfunction and urinary incontinence.
\end{abstract}

Keywords: Quality of life, Pelvic floor, Pregnancy, Nursing.

\section{RESUMEN}

Objetivo: Comparar la calidad de vida relacionada con la disfunción del piso pélvico en mujeres embarazadas atendidas en el sistema de salud público y privado, así como identificar la prevalencia de la disfunción del piso pélvico en mujeres embarazadas de bajo riesgo atendidas en estos escenarios. Métodos: Estudio transversal con una muestra de 261 mujeres embarazadas y la recolección se realizó de septiembre a enero de 2015. Los participantes recibieron un formulario de consentimiento y se respetaron los principios de la bioética. Resultados: Los datos mostraron que las mujeres embarazadas atendidas en el sistema público tienen casi 4 veces más probabilidades de tener disfunción sexual en comparación con las mujeres embarazadas en la atención privada. Las mujeres embarazadas en atención privada tuvieron mejor QVRS en todos los dominios. Aunque las mujeres embarazadas del servicio privado son menos activas

\footnotetext{
${ }^{1}$ Universidade Federal do Ceará (UFC), Fortaleza - CE. *E-mail: paularenatal@yahoo.com.br
}

2 Universidade Regional do Cariri (URCA), Crato - CE. 
sexualmente, los resultados mostraron que estas mujeres tienden a tener menos disfunción sexual, con asociación significativa en todos los dominios. Los dominios que tuvieron el mayor impacto negativo en el orden creciente de los promedios en los dos servicios fueron similares, siendo: "deseo ", "arousal", "orgimento"," lubricación ", "dolor" y "satisfacción ". Los dominios "lubricación" y "dolor" en el servicio privado presentaban medios idénticos. Conclusión: Se concluye que las mujeres embarazadas seguidas en el servicio privado tuvieron mejores índices de calidad de vida en general, asociados con disfunción sexual e incontinencia urinaria.

Palavras-chave: Calidad de vida, Diafragma pélvico, Embarazo, Enfermería.

\section{INTRODUÇÃO}

O termo Disfunção do Assoalho Pélvico (DAP) engloba uma larga variedade de condições clínicas interrelacionadas, que inclui: disfunção do trato urinário inferior, prolapso de órgãos pélvicos, disfunção defecatória, disfunção sexual e vários distúrbios de dor pélvica (WALTERS MD e KARRAM MM, 2016).

Dentre as principais DAP encontram-se, a Incontinência Urinária (IU), que é definida como a perda involuntária de urina e a disfunção sexual que se refere a qualquer modificação relacionada a resposta sexual humana que produz sofrimento físico e/ou emocional, individual ou entre parceiros (HAYLEN B, et al., 2010; ORGANIZAÇÃO MUNDIAL DA SAÚDE, 1993). Destaca-se que tais disfunções atingem cerca de 35,5\% das mulheres e que prejudicam significativamente a produtividade e a Qualidade de Vida (QV) de mulheres na vida adulta (LEMOS AQ e SÁ KN, 2017).

Nessa conjuntura, as DAP configuram-se como condições que podem comprometer a QV das mulheres, principalmente no período gestacional. A incontinência tem relação com baixos indicadores de saúde física e mental, além de afetar a capacidade funcional, agravar ou desencadear a depressão, déficit cognitivo e autopercepção de saúde como péssima/ruim (KESSLER M, et al., 2018). Já a disfunção sexual, por sua vez, também acarreta sentimento de impotência e baixa autoestima, impactando negativamente a relação entre 0 casal (ABDO CHN e FLEURY HJ, 2006).

Assim, durante o período gestacional, tais disfunções devem ser avaliadas, no intuito de evitar danos permanentes e que possam acarretar prejuízos na QV das mulheres. Essas disfunções associadas ao período de mudanças característico da gestação potencializam o comprometimento da QV (OLIVEIRA SG, et al., 2021).

Deste modo, um dos momentos oportunos para uma avaliação das disfunções sexuais entre gestantes é durante o acompanhamento nas consultas de pré-natal. Sabe-se que na conjuntura brasileira, há diferentes classes econômicas, o que interfere diretamente na prestação do serviço de saúde no pré-natal, pois algumas gestantes são atendidas no serviço público e outras no serviço privado. A despeito de existirem tais modalidades de atendimentos, deve-se alertar para que a qualidade do atendimento seja semelhante, garantindo uma avaliação integral da mulher, que inclua aspectos relacionados à QV e, também, cuidados direcionados às disfunções do assoalho pélvico, sem quaisquer distinções na qualidade da assistência em saúde prestada (CESAR JA, et al., 2012).

Nesse contexto, diante das diversas repercussões na vida da gestante, medidas de avaliação da QV relacionadas com a disfunção do assoalho pélvico necessitam ser implementadas, pois poderão evidenciar problemas relacionados ao assoalho pélvico, além de esclarecer se existem diferenças significativas entre 0 grupo de mulheres atendidas no sistema público e privado (CESAR JA, et al., 2012).

Isto posto, o presente artigo teve como objetivo comparar a QV relacionada à disfunção do assoalho pélvico entre gestantes atendidas no sistema público e privado de saúde, bem como identificar a prevalência das DAP em gestantes atendidas nesses dois cenários.

\section{MÉTODOS}

Trata-se de um estudo transversal, realizado em três unidades básicas de saúde que atendem gestantes pelo Sistema Único de Saúde (SUS) e em uma clínica que atende pelo sistema particular que oferece serviços na área obstétrica e ginecológica.

A população da pesquisa se foram gestantes de baixo risco. Já a amostra foi composta por 261 gestantes, sendo 120 do serviço privado e 141 do público. O cálculo da amostra foi feito utilizando a fórmula para 
populações finitas, na qual o $\mathrm{N}$ foi obtido por meio do somatório de todas as consultas mensais das gestantes atendidas nos locais de pesquisa selecionados, o que findou em 800 consultas. Considerou-se o nível de confiança de $95 \%$, o erro máximo permitido de $5 \%$ e a porcentagem com a qual o fenômeno se verifica e a porcentagem complementar foi de $50 \%$.

Os critérios de inclusão foram gestantes em acompanhamento no pré-natal risco habitual e serem alfabetizadas, pois complicações de um pré-natal de alto risco podem interferir na QV relacionada à disfunção do assoalho pélvico e foi necessário o letramento para responder ao questionário de QV de Ferrans e Powers adaptado.

A coleta de dados ocorreu entre setembro de 2014 a janeiro de 2015. As gestantes foram convidadas a participar da pesquisa enquanto aguardavam atendimento para a consulta de acompanhamento pré-natal no SUS ou enquanto esperavam a realização de exames obstétricos no sistema privado. Após o aceite, as participantes foram encaminhadas às salas reservadas para preenchimento do Termo de Consentimento Livre e Esclarecido (TCLE) e aplicação dos instrumentos de pesquisa, mantendo a privacidade das respostas fornecidas.

Foram utilizados os seguintes instrumentos de coleta de dados: 1) um questionário contendo os fatores sociodemográficos, obstétricos e comportamentais; 2) o questionário Índice de Qualidade de Vida de Ferrans \& Powers adaptado e validado para avaliar a QV das gestantes; 3) o Female Sexual Function Index (FSFI) validado no Brasil para avaliar a função sexual das gestantes e; 4) o instrumento King's Health Questionnaire (KHQ) para investigar a QV de mulheres com incontinência urinária também validado (OLIVEIRA AS e SANTOS VLCG, 2012; FONSECA ESM, et al., 2005).

O instrumento KHQ é composto por 21 questões e possui 9 domínios: 1) Percepção geral de saúde, 2) Impacto da incontinência urinária, 3) Limitações de atividades diárias, 4) Limitações físicas, 5) Limitações sociais, 6) Relacionamento pessoal, 7) Emoções, 8) Sono/disposição, 9) Medidas de gravidade. O instrumento é pontuado por cada um de seus domínios, variando de 0 a 100, quando maior a pontuação obtida, pior é a QVRS referente aquele domínio. Ressalta-se que o KHQ foi aplicado somente para àquelas mulheres que verbalizavam queixa de perda urinária, o que correspondeu a 68 gestantes. Por outro lado, o FSFI foi aplicado com 190 grávidas que relataram estarem sexualmente ativas nas últimas quatro semanas, condição imposta pelas autoras da escala para investigação da função sexual.

As variáveis coletadas no questionário dos fatores sociodemográficos, obstétricos e comportamentais foram: idade, estado civil, escolaridade, renda familiar, se possui trabalho remunerado, religião, trimestre gestacional, Índice de Massa Corporal (IMC), paridade, tipo de parto, idade gestacional da primeira consulta, planejamento da gestação, apoio do parceiro, recebimento de orientações educativas sobre DAP na gestação, prática de atividade física e uso de drogas lícitas e ilícitas.

O instrumento FSFI foi utilizado para avaliar a disfunção sexual e as dimensões principais da função sexual da mulher, composta por cinco domínios da resposta sexual: 1) Desejo e estímulo subjetivo, 2) Lubrificação, 3) Orgasmo, 4) Satisfação e 5) Dor ou desconforto. A pontuação final da escala pode variar de 2 a 36 , sendo obtida pela soma dos escores ponderados em relação a cada domínio. Para caracterizar disfunção sexual, o ponto de corte deverá ser abaixo de 26,5.

Os dados foram compilados e analisados por meio do programa estatístico Statistical Package for the Social Sciences (SPSS), versão 20.0. Calcularam-se medianas e desvios-padrão das variáveis quantitativas. Foram feitos a priori os testes de Levene para verificar a homogeneidade das variâncias; e o teste de Kolmogorov-Smirnov para testar a normalidade das variáveis. Na sequência, as médias da escala FSFI e $\mathrm{KHQ}$ foram analisadas pelos testes $t$ de student, Mann-Whitney e Wilcoxon.

As associações entre as variáveis e a disfunção sexual foram feitas utilizando o teste de $\chi 2$, e calculou-se a Razão de Chances (RC) e seu respectivo intervalo de confiança de 95\% (IC95\%), quando se obteve o $p$ significativos. As análises inferenciais foram consideradas estatisticamente significantes se $p<0,05$.

A pesquisa foi aprovada pelo Comitê de Ética e Pesquisa (CEP) da Maternidade Escola Assis Chateaubriand (MEAC) da Universidade Federal do Ceará (UFC) sob o parecer de ํㅜ 770.902. 


\section{RESULTADOS}

Primeiramente seguem os dados de caracterização da amostra de forma separada, correspondendo aos serviços públicos e privados dispostos na Tabela 1.

Tabela 1 - Distribuição das variáveis sociodemográficas, obstétricas e comportamentais de gestantes atendidas no sistema público e privado, 2015.

\begin{tabular}{|c|c|c|c|c|c|c|}
\hline \multirow[t]{2}{*}{ Variáveis } & \multicolumn{2}{|c|}{$\begin{array}{c}\text { Sistema Público } \\
\text { Possui DAP }\end{array}$} & \multicolumn{3}{|c|}{$\begin{array}{c}\text { Sistema Privado } \\
\text { Possui DAP }\end{array}$} & \multirow{2}{*}{$p$ value } \\
\hline & Sim & Não & p value & Sim & Não & \\
\hline \multicolumn{7}{|l|}{ Idade } \\
\hline $\begin{array}{l}\text { Até } 35 \text { anos } \\
35 \text { anos ou mais }\end{array}$ & $\begin{array}{c}91 \\
3\end{array}$ & $\begin{array}{c}45 \\
2\end{array}$ & 0,747 & $\begin{array}{l}52 \\
11\end{array}$ & $\begin{array}{c}48 \\
9\end{array}$ & 0,806 \\
\hline \multicolumn{7}{|l|}{ Estado Civil } \\
\hline Com parceiro & 78 & 36 & 0,364 & 62 & 55 & 0,604 \\
\hline \multirow{2}{*}{\multicolumn{7}{|c|}{ Escolaridade }} \\
\hline $\begin{array}{l}\text { Escolarıdade } \\
\text { Até } 9 \text { anos de estudo }\end{array}$ & & & & & & \\
\hline 9 ou mais anos de estudo & $\begin{array}{l}35 \\
59\end{array}$ & 18 & 0,902 & 5 & 6 & 0,623 \\
\hline \multicolumn{7}{|l|}{ Trabalha fora } \\
\hline Sim & 32 & 16 & 1,000 & 55 & 51 & 0,711 \\
\hline Não & 62 & 31 & & 8 & 6 & \\
\hline \multicolumn{7}{|l|}{ Renda familiar (SM) } \\
\hline Até 1 salário & 38 & 15 & 0,325 & 3 & 1 & 0,621 \\
\hline 1 salário ou mais & 56 & 32 & & 60 & 56 & \\
\hline \multicolumn{7}{|l|}{ Religião } \\
\hline Com religião & 86 & 42 & 0,681 & 59 & 55 & 0,682 \\
\hline Sem religião & 8 & 5 & & 4 & 2 & \\
\hline \multicolumn{7}{|c|}{ Dados obstétricos Trimestre gestacional $(\mathrm{n}=261)$} \\
\hline $1^{\circ}$ trimestre & 16 & 8 & 0,922 & 12 & 8 & 0,574 \\
\hline $2 \circ$ trimestre & 49 & 23 & & 25 & 28 & \\
\hline $3 \circ$ trimestre & 29 & 16 & & 25 & 21 & \\
\hline \multicolumn{7}{|c|}{ Classificação do IMC/Semana gestacional $(\mathbf{n}=257)$} \\
\hline Baixo peso & 56 & 25 & 0,470 & 32 & 27 & 0.708 \\
\hline Adequado & 38 & 22 & & 31 & 30 & \\
\hline \multicolumn{7}{|l|}{ Paridade $(\mathrm{n}=261)$} \\
\hline Nulípara & 57 & 25 & 0,398 & 32 & 34 & 0,330 \\
\hline Multípara & 37 & 22 & & 31 & 23 & \\
\hline \multicolumn{7}{|l|}{ Tipo de parto $(n=113)$} \\
\hline Vaginal & 16 & 14 & 0,071 & 4 & 2 & 1,000 \\
\hline Abdominal & 22 & 7 & & 27 & 21 & \\
\hline \multicolumn{7}{|c|}{ Início do acompanhamento gestacional $(n=261)$} \\
\hline$<12$ semanas & 52 & 33 & 0,088 & 61 & 55 & 0,652 \\
\hline$\geq 12$ semanas & 42 & 14 & & 2 & 2 & \\
\hline \multicolumn{7}{|c|}{ Fatores comportamentais $(\mathrm{n}=261)$} \\
\hline \multicolumn{7}{|c|}{ Gravidez planejada } \\
\hline Sim & 32 & 17 & 0,803 & 52 & 42 & 0,240 \\
\hline Não & 62 & 30 & & 11 & 15 & \\
\hline \multicolumn{7}{|l|}{ Apoio do parceiro } \\
\hline Sim & 85 & 44 & 0,522 & 63 & 57 & - \\
\hline Não & 9 & 3 & & 0 & 0 & \\
\hline Orientação educativa dur & & & & & & \\
\hline Sim & 36 & 26 & & 23 & 21 & 0,970 \\
\hline Não & 58 & 21 & 0,06 & 40 & 36 & \\
\hline Pratica atividade física & & & & & & \\
\hline Sim & 4 & 7 & 0,042 & 15 & 9 & 0,273 \\
\hline Não & 90 & 40 & & 48 & 48 & \\
\hline Uso de cigarro & & & & & & \\
\hline Sim & 2 & 2 & 0,365 & 1 & 0 & 0,525 \\
\hline Não & 93 & 45 & & 62 & 57 & \\
\hline Uso de Álcool & & & & & & \\
\hline Sim & 1 & 2 & 0,258 & 63 & 57 & - \\
\hline Não & 93 & 45 & & 0 & 0 & \\
\hline Uso de droga ilícita & & & & & & \\
\hline Sim & 1 & 0 & 1,000 & 63 & 57 & - \\
\hline Não & 93 & 47 & & 0 & 0 & \\
\hline
\end{tabular}

Fonte: Soares PRAL, et al., 2022. 
A mediana de idade mostrou grande diferença entre gestantes atendidas no serviço público e privado apresentando respectivamente 23 e 32 . No que se refere aos anos de estudo, gestantes do serviço privado apresentaram uma mediana de 16 em comparação com 11 do sistema público. Já a renda foi a variável sociodemográfica com maior discrepância sendo $R \$ 8.000,00$ do serviço privado e $R \$ 1.100,00$ do público. Quanto ao início do acompanhamento do pré-natal, na rede privada se deu com seis semanas, enquanto na rede pública, se iniciou com 12 semanas.

A análise das variáveis sociodemográficas, obstétricas e comportamentais não revelaram associação estatisticamente significativa quando comparadas os serviços de atendimento de saúde, com exceção da prática de atividade física no pré-natal, em que gestantes atendidas no serviço privado praticavam $25 \%$ mais exercícios na gestação do que gestantes da rede pública.

A Tabela 2 comparou a QVRS de gestantes acompanhadas pela rede pública e privada.

Tabela 2 - Comparação do índice de QV de Ferrans \& Powers entre gestantes atendidas nos serviços públicos e privados, 2014.

\begin{tabular}{lccc}
\hline Domínios & $\begin{array}{c}\text { Público } \\
\text { Média } \pm \text { DP }\end{array}$ & $\begin{array}{c}\text { Privado } \\
\text { Média } \pm \text { DP }\end{array}$ & Valor de p \\
\hline D1- Saúde & $21,6 \pm 4,0$ & $22,5 \pm 3,3$ & 0,051 \\
D2- Sócio econômico & $21,1 \pm 5,3$ & $24,9 \pm 3,3$ & 0,000 \\
D3- Psicológico & $25,1 \pm 5,0$ & $26,4 \pm 2,7$ & 0,013 \\
D4- Família & $27,0 \pm 3,9$ & $27,9 \pm 2,5$ & 0,028 \\
D- Total & $22,8 \pm 3,7$ & $24,4 \pm 2,6$ & 0,000 \\
\hline
\end{tabular}

Fonte: Soares PRAL, et al., 2022.

A análise das médias acerca da QV foi estatisticamente significativa em todos os domínios, com exceção do domínio "Saúde", evidenciando que gestantes atendidas no serviço privado possuem melhor QV no que se refere aos aspectos socioeconômicos, psicológicos, familiares e de forma geral.

A Tabela 3 apresentou a distribuição das disfunções do assoalho pélvico mais frequentes no estudo, que foram a disfunção sexual e a IU.

Tabela 3 - Distribuição das disfunções do assoalho pélvico em gestantes atendidas no sistema público e privado, 2015.

\begin{tabular}{|c|c|c|c|c|c|c|c|}
\hline \multirow{2}{*}{ DAP $(n=261)$} & \multicolumn{2}{|c|}{ Público } & \multicolumn{2}{|c|}{ Privado } & \multirow{2}{*}{ Valor de $p$} & \multirow{2}{*}{ RC } & \multirow{2}{*}{ IC } \\
\hline & $\mathbf{N}$ & $\%$ & $\mathbf{N}$ & $\%$ & & & \\
\hline \multicolumn{8}{|c|}{ Disfunção sexual } \\
\hline Sim & 49 & 42,2 & 12 & 16,2 & 0,000 & 3,8 & $1,8-7,7$ \\
\hline Não & 67 & 57,8 & 62 & 83,3 & & - & - \\
\hline \multicolumn{8}{|c|}{ Incontinência Urinária } \\
\hline Sim & 38 & 27 & 24 & 20 & 0,189 & - & - \\
\hline Não & 103 & 73 & 96 & 80 & & & \\
\hline
\end{tabular}

Fonte: Soares PRAL, et al., 2022.

Os dados evidenciaram que $31,2 \%$ (38) das mulheres que apresentaram IU foram atendidas no sistema público e $18,3 \%$ (24) no serviço privado. No que se refere a disfunção sexual, analisando a amostra total, observou-se que $27,2 \%$ (71) das gestantes verbalizaram que não tiveram relação sexual nas últimas quatro semanas. Percebeu-se ainda que do total da amostra (141) de gestantes do serviço público, 116 (82,3\%) relataram ter relação sexual nas últimas quatro semanas. Por sua vez, no serviço privado essa porcentagem caiu para $61,6 \%$ (74) de gestantes que permaneceram ativas sexualmente no último mês.

Os resultados apontaram que gestantes atendidas no serviço público tem quase quatro vezes mais chances de ter disfunção sexual, quando comparadas com gestantes da assistência privada. Já a frequência de IU não revelou diferença significativa entre os grupos comparados.

A Tabela 4 avaliou a QV de gestantes com IU atendidas nos serviços públicos e privados. 
Tabela 4 - Influência do sistema de acompanhamento pré-natal na QVRS em gestantes com IU, 2014

\begin{tabular}{lccc}
\hline DOMiNIOS & Público* $^{*}(\mathbf{n}=\mathbf{4 4})$ & Privado* $^{*}(\mathbf{n}=\mathbf{2 2})$ & Valor de $\mathbf{p}$ \\
\hline Percepção geral da saúde & $28,9 \pm 21,5$ & $14,7 \pm 16,6$ & 0,002 \\
Impacto da incontinência & $27,2 \pm 28,9$ & $0,0 \pm 0,0$ & 0,000 \\
Limitação das atividades diárias & $20,4 \pm 27,8$ & $0,0 \pm 0,0$ & 0,000 \\
Limitações físicas & $15,9 \pm 29,8$ & $0,0 \pm 0,0$ & 0,004 \\
Limitações sociais & $8,4 \pm 19,4$ & $0,0 \pm 0,0$ & 0,006 \\
Relações pessoais & $5,3 \pm 17,1$ & $0,0 \pm 0,0$ & 0,039 \\
Emoções & $10,3 \pm 22,2$ & $0,0 \pm 0,0$ & 0,004 \\
Sono & $15,5 \pm 28,3$ & $0,0 \pm 0,0$ & 0,003 \\
Medida de gravidade & $12,2 \pm 21,3$ & $0,9 \pm 4,3$ & 0,001
\end{tabular}

Legenda: *Média e desvio padrão. Fonte: Soares PRAL, et al., 2022.

As gestantes atendidas no serviço privado tiveram melhor QVRS em todos os domínios, com significância estatística. Além disso, os dados revelam que em quase todos os domínios essas mulheres não sofreram nenhum impacto em sua QVRS, com exceção do domínio "Percepção" e "Medida de gravidade", mesmo assim com médias baixas.

A Tabela 5 apresenta as médias da função sexual de gestantes atendidas no serviço público e privado.

Tabela 5 - Comparação entre as médias dos domínios da função sexual entre o serviço público e privado, 2014.

\begin{tabular}{lccc}
\hline DOMíNIOS & Público* $^{*}(\mathbf{n = 1 1 6})$ & Privado* $^{*}(\mathbf{n}=\mathbf{7 4})$ & Valor de $\mathbf{p}$ \\
\hline Desejo & $3,4 \pm 1,1$ & $3,6 \pm 1,0$ & 0,174 \\
Excitação & $3,9 \pm 1,0$ & $4,5 \pm 0,9$ & 0,000 \\
Lubrificação & $4,8 \pm 1,0$ & $5,2 \pm 0,9$ & 0,045 \\
Orgasmo & $4,1 \pm 1,3$ & $4,7 \pm 1,1$ & 0,002 \\
Satisfação & $5,0 \pm 1,1$ & $5,4 \pm 0,8$ & 0,025 \\
Dor & $4,9 \pm 1,1$ & $5,2 \pm 1,1$ & 0,023 \\
Escala Total & $26,3 \pm 0,8$ & $28,6 \pm 0,7$ & 0,001 \\
\hline
\end{tabular}

Legenda: *Média e desvio padrão. Fonte: Soares PRAL, et al., 2022.

Embora às gestantes do serviço privado sejam menos ativas sexualmente, os resultados demonstraram que em todos os domínios acima, as mesmas apresentaram maiores médias em comparação com as do serviço público, apontando que elas tendem a ter menos disfunção sexual, com associação significativa em todos os domínios.

Os domínios que apresentaram maior impacto negativo na ordem crescente de médias no serviço público e privado foram semelhantes sendo: "Desejo", "Excitação", "Orgasmo", "Lubrificação", "Dor" e "Satisfação". Ressalta-se que os domínios "Lubrificação" e "Dor" no serviço privado apresentaram médias idênticas de 5,2.

\section{DISCUSSÃO}

Discorrendo sobre os dados do perfil da amostra das gestantes atendidas nos serviços públicos e privados observou-se grandes diferenças quanto a idade das mulheres, a escolaridade e a renda. Estes achados vão de encontro com perfil encontrado em pesquisa realizada no Rio Grande do Sul, em que as mulheres atendidas no serviço público eram, em média, três anos mais novas, tinham três anos a menos de escolaridade e viviam com cerca de metade da renda das mulheres atendidas no serviço privado (CESAR JA, et al., 2012).

Percebe-se que a mulher atendida no serviço público pode vivenciar contextos socioeconômicos mais desfavoráveis, o que pode comprometer outros aspectos da QV, incluindo uma maior dificuldade de acesso ao serviço de saúde com acolhimento adequado e no tempo oportuno para resolutividade de suas demandas de cuidado (CESAR JA, et al., 2012).

Os distúrbios do assoalho pélvico podem afetar adversamente a QV das mulheres e podendo ocorrer durante diferentes fases da vida feminina, ampliando durante e após a gravidez (YOHAY D, et al., 2016; HUEBNER $\mathrm{M}$, et al., 2010). 
Segundo um estudo de coorte realizado na Austrália, o controle de peso durante a gravidez, sobretudo com a prática de atividades físicas, é parte importante no aconselhamento durante a gestação, visto que a obesidade é um fator de risco significativo para vários distúrbios do assoalho pélvico (BODNER-ADLER $B$, et al., 2019). No presente estudo, que as gestantes atendidas no serviço privado praticavam $25 \%$ mais exercícios na gestação do que gestantes da rede pública, o que evidencia que apesar de ser importante o aconselhamento da prática de atividades físicas, existe uma discrepância na realização das atividades nesses dois serviços analisados.

Quanto ao início do acompanhamento do pré-natal, na rede privada se deu com 6 semanas e com 12 na rede pública, mostrando que, no que se refere a esse aspecto, obtém-se maior qualidade no serviço privado. Tais resultados podem ser explicados por diversos aspectos sendo eles: procura precoce das gestantes ao serviço privado, maior planejamento por parte dessas mulheres e início precoce do serviço ao primeiro sinal de gestação, bem como a falta de vagas para atendimento de pré-natal no serviço público. Diante disso, gestantes do serviço público podem ter comprometimento na suplementação de ácido fólico prescrita até o fim do primeiro trimestre (BRASIL, 2016).

Ademais é válido ressaltar que o período adequado de acompanhamento é importante para que as mulheres recebam uma melhor assistência, pois segundo um estudo observacional realizado em São Paulo, a identificação precoce das disfunções do assoalho pélvico é importante para que se oriente adequadamente as mulheres durante o pré-natal, visto que muitas delas podem perceber essas disfunções como fisiológicas e inerentes ao processo da gravidez, adiando e dificultando o tratamento de forma adequada, prejudicando a saúde física e emocional da mulher (OLIVEIRA SMJV, et al., 2018).

$\mathrm{Na}$ avaliação da QV das gestantes nos dois grupos, encontrou-se associação estatisticamente significativas, nos domínios "Socioeconômico", "Psicológico", "Familiar" e "Total". Destaca-se que a renda apresentou discrepância, sendo no serviço privado 7 vezes maior do que a do serviço público. Esses dados corroboram com estudos que afirmam que o aumento da condição socioeconômica possibilita acesso a melhor nutrição, atividade física e maior acesso à assistência à saúde, o que implica diretamente em uma melhor QV (BATISTA EM, et al., 2011).

O domínio psicológico com maior pontuação entre as mulheres atendidas no serviço privado, pode ser explicado pelo fato destas possuírem melhor QV também nos aspectos socioeconômico, familiar e de forma geral, uma vez que todas essas dimensões corroboram para um melhor equilíbrio psicossocial. Este entendimento foi igualmente demonstrado em estudo realizado em Campinas-SP em que mulheres com maior escolaridade, sem problemas atuais de saúde e boa coesão familiar possuem melhor QV (ROSALINI MP, et al., 2019).

Já no domínio familiar, ressalta-se a importância do suporte social para a QV das gestantes, visto que esse apoio possibilita o enfrentamento das dificuldades durante a gravidez e, como pode ser observado no estudo com mulheres grávidas no Paquistão, em que o suporte social e QV apresentam uma relação positiva (GUL B, et al., 2018).

Compreende-se a importância do suporte familiar quando se considera também os resultados apresentados em pesquisa realizada em Vitória-ES, em que a situação conjugal insegura e os conflitos familiares podem ser relacionados a gestações de alto risco (GARCIA EM, et al., 2019). Desta maneira, reforça-se o impacto positivo que o apoio familiar pode proporcionar na QV das gestantes.

Analisando os dados relacionados à DAP, os resultados dessa pesquisa apontaram que gestantes atendidas no serviço público tem quase quatro vezes mais chances de ter disfunção sexual, quando comparadas com gestantes da assistência privada. As evidências de um estudo multicêntrico realizado com mulheres com distúrbios do assoalho pélvico nos Estados Unidos e Reino Unido mostrou que mulheres sexualmente ativas eram mais propensas a ter um assoalho pélvico mais forte (KANTER G, et al., 2015).

Contrariando essas evidências, no presente estudo, as gestantes do serviço público 116 (82,3\%) foram as mais ativas sexualmente em comparação com às do serviço privado, entretanto, estas tiveram melhor QVRS 
em todos os domínios, além de possuírem menor prevalência de disfunção sexual. Esses dados demonstram que às DAP podem sofrer influência não somente do aspecto fisiológico, mas também se faz necessário avaliar o impacto das dimensões socioeconômicas, familiares e psicológicas no desenvolvimento de um desfecho clínico desfavorável associado às DAP nessas mulheres.

Por sua vez, a saúde sexual feminina pode sofrer impactos negativos por meio de disfunções do assoalho pélvico. O grau de implicação destes sintomas pode comprometer a adequada expressão de sua sexualidade, levando as mesmas a vivenciarem situações como isolamento ou mudanças na auto percepção (YOUNT SM, 2013).

Estudo realizado em São Paulo apontou que a maioria das gestantes atendidas em um serviço público referem função sexual considerada entre regular a excelente (64\%), o que está relacionado à QV e à satisfação sexual (FERREIRA DQ, et al., 2012). Neste sentido, este dado reflete o que foi encontrado na presente pesquisa no que diz respeito ao fato das mulheres atendidas no serviço público serem mais ativas sexualmente.

A incontinência urinária apresentou impactos negativos na QV das mulheres atendidas no sistema público, sob a ótica de diversos domínios. Este achado é corroborado com um estudo realizado na Turquia, pois apresentou que a incontinência urinária pode afetar a QV da mulher no aspecto do seu bem-estar psicológico e social. Apesar desse estudo não se tratar de gestantes, o estudo demonstra essa percepção das mulheres em considerar a IU um problema de saúde que interfere na QV (TURKCU SG e KUKULU K, 2017).

Um outro estudo realizado em Sergipe comparou a QV de gestantes com e sem perda urinária e identificou que a perda urinária reduz a QV das gestantes, resultando em uma piora da percepção geral de saúde e impacto da incontinência ao final da gestação (MOCCELLIN AS, et al., 2014). Desta forma, compreende-se que as mulheres atendidas no serviço público sofrem mais efeitos negativos na sua QV durante a gestação em decorrência de IU.

Assim, investigar a QV de gestantes associada ao desenvolvimento das DAP nos diferentes serviços de saúde podem auxiliar na diminuição de desfecho desfavorável para essas mulheres, visto que as DAP comprometem a QV das gestantes, permeando diversos aspectos que abordam tanto os domínios psicológicos, socioeconômicos, como familiares.

\section{CONCLUSÃO}

As mulheres atendidas na rede particular apresentaram melhor QV sob aspectos relacionados à prática adequada de atividade física e possuem menor chance de sofrerem alguma disfunção sexual quando comparadas às mulheres atendidas na rede pública, muito embora estas tenham sido mais ativas sexualmente no mês anterior à pesquisa. Evidencia-se que as gestantes acompanhadas no ambiente privado apresentaram melhores resultados no domínio relacionado à QV sexual e apresentam menor incidência de incontinência urinária durante o pré-natal. Dessa forma, mulheres do serviço público merecem mais atenção quanto à avaliação da $\mathrm{QV}$ associada às DAP, especialmente no que se refere às incontinências urinárias e QV sexual.

\section{REFERÊNCIAS}

1. ABDO CHN, et al. Aspectos diagnósticos e terapêuticos das disfunções sexuais femininas. Arch. Clin. Psychiatry, 2006, 33(3): 162-167.

2. BATISTA EM, et al. Comparison of pelvic floor muscle strength between women undergoing vaginal delivery, cesarean section, and nulliparae using a perineometer and digital palpation. Gynecol Endocrinol, 2011; 27(11): 910-914.

3. BODNER-ADLER B, et al. Prevalence and risk factors for pelvic floor disorders during early and late pregnancy in a cohort of Austrian women. Arch Gynecol Obstet, 2019; 300: 1325-30.

4. BRASIL. Protocolos da Atenção Básica: Saúde das Mulheres. 2016. Disponível em: https://bvsms.saude.gov.br/bvs/publicacoes/protocolos_atencao_basica_saude_mulheres.pdf. Acesso em: $16 \mathrm{de}$ janeiro de 2022. 
5. CESAR JA, et al. Assistência pré-natal nos serviços públicos e privados de saúde: estudo transversal de base populacional em Rio Grande, Rio Grande do Sul, Brasil. Cad. Saúde Pública, 2012; 28(11): 2106-2114.

6. FERREIRA DQ, et al. Função sexual e qualidade de vida em gestantes de baixo risco. Rev. Bras. Ginecol. Obstet, 2012; 34(9): 409-413.

7. FONSECA ESM, et al. Validação do questionário de qualidade de vida (King's Health Questionnaire) em mulheres brasileiras com incontinência urinária. Rev. Bras. Ginecol. Obstet, 2005; 27(5): 235-242.

8. GARCIA EM. Risco gestacional e desigualdades sociais: uma relação possível?. Ciênc. Saúde Coletiva, 2019; 24(12): 4633-4642.

9. GUL B, et al. Social support and health related quality of life among pregnant women. JPMA, 2018; 68(6): 872-875.

10. HAYLEN B, et al. An International Urogynecological Association (IUGA)/International Continence Society (ICS) joint report on the terminology for female pelvic floor dysfunction. Int Urogyn J, 2010; 21(1): 5-26.

11. HUEBNER $\mathrm{M}$, et al. The impact of pregnancy and vaginal delivery on urinary incontinence. Int $\mathrm{J}$ Gynaecol Obstet, $2010 ; 110(3):$ 249-251.

12. KANTER G, et al. A strong pelvic floor is associated with higher rates of sexual activity in women with pelvic floor disorders. Int Urogynecol J, 2015; 26(7): 991-996.

13. KESSLER M, et al. Prevalência de incontinência urinária em idosos e relação com indicadores de saúde física e mental. Rev. Bras. Geriatr. Gerontol, 2018; 21(4): 397-407.

14. LEMOS $A Q$, SÁ KN. Parâmetros angulares da pelve e a função muscular do assoalho pélvico em mulheres com incontinência urinária. Dissertação (Mestrado em Saúde Pública). Escola Bahiana de Medicina e Saúde Pública, Salvador, 2017;101 p.

15. MOCCELLIN AS, et al. Incontinência urinária na gestação: implicações na qualidade de vida. Rev. Bras. Saude Mater. Infant, 2014; 14(2): 147-154.

16. OLIVEIRA AS, SANTOS VLCG. Índice de qualidade de vida de Ferrans \& Powers - versão feridas: estudo da responsividade. Tese (Doutorado em Enfermagem). Escola de Enfermagem, São Paulo, 2012; 116 p.

17. OLIVEIRA SG, et al. Disfunções do assoalho pélvico em primíparas até 6 meses após o parto: estudo de corte. Rev Bras Enferm, 2021; 74(5):e20200607.

18. OLIVEIRA SMJV, et al. Disfunciones del suelo pélvico en mujeres primíparas después del parto. Enfermería Global, $2018 ; 17(3): 26-67$.

19. ORGANIZAÇÃO MUNDIAL DA SAÚDE. Classificação de Transtornos Mentais e de Comportamento da CID-10. Descrições Clínicas e Diretrizes Diagnósticas. Porto Alegre: Artmed, 1993; 352p.

20. ROSALINI MP, et al. Qualidade de vida, coesão e adaptabilidade em famílias beneficiárias do Programa Bolsa Família. Ciência \& Saúde Coletiva, 2019; 24(1): 307-314.

21. TÜRKCÜ SG, KUKULU K. Urinary incontinence and quality of life of women living in nursing homes in the Mediterranean region of Turkey. Psychogeriatrics, 2017; 17(6): 446-452.

22. WALTERS MD, KARRAM MM. Uroginecologia e Cirurgia Reconstrutiva Pélvica. 4 ed. São Paulo: Elsevier, 2016; 688p.

23. YOHAY D, et al. Prevalence and trends of pelvic floor disorders in late pregnancy and after delivery in a cohort of Israeli women using the PFDI-20. Eur J Obstet Gynecol Reproduct Biol, 2016; 200: 35-39.

24. YOUNT SM. The Impact of Pelvic Floor Disorders and Pelvic Surgery on Women's Sexual Satisfaction and Function. Journal of Midwifery \& Women's Health, 2013; 58(5): 538-545. 